\title{
COMBINED EFFECT OF CHEMICAL ENHANCERS AND ULTRASOUND ON THE SKIN PERMEATION OF CAFFEINE
}

Thanaa M. E. Borg

Department of Pharmaceutics, Faculty of Pharmacy, Mansoura University, Mansoura, Egypt

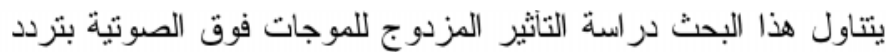

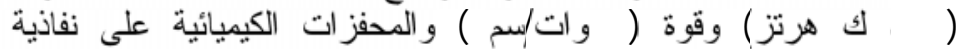

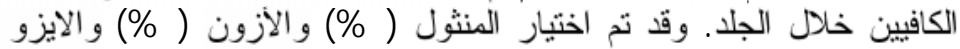

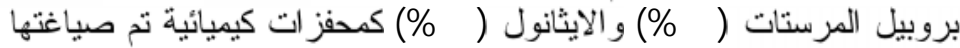

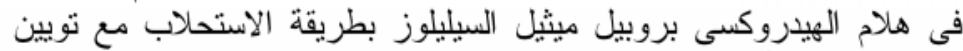

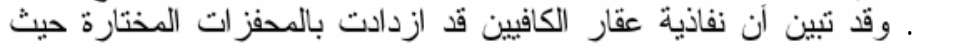
كانت قيم التنفق

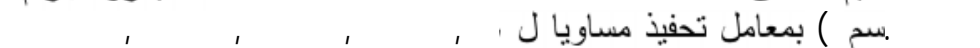

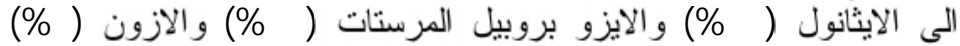

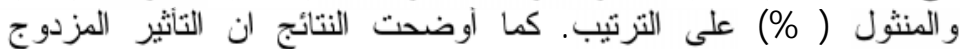

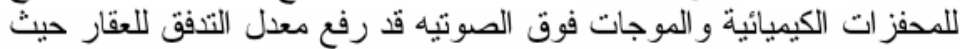

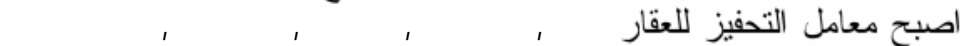

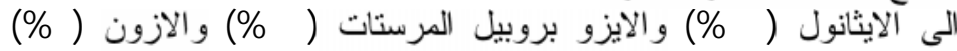

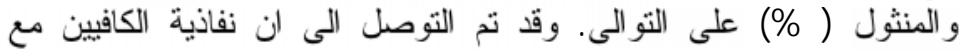

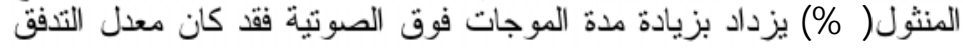

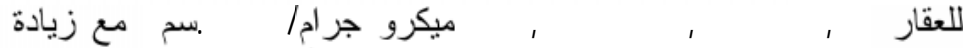

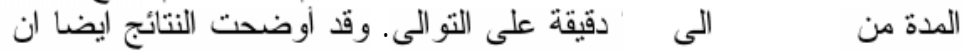

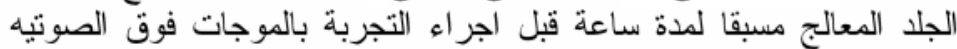

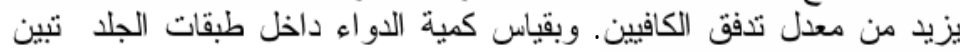

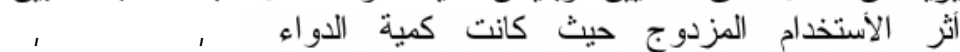

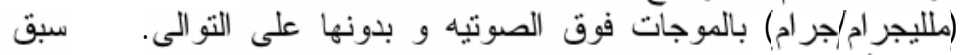

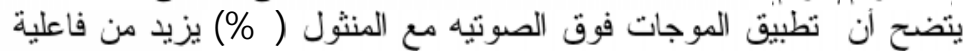
العلاج عبر الجلد و العلاج الموضعى لعقار الكافيين.

The combined effect of $800 \mathrm{kHz}$ ultrasound with $2 \mathrm{~W} / \mathrm{cm}^{2}$ intensity and chemical enhancers on the skin permeation of caffeine was investigated using excised ear rabbit skin. Menthol, laurocapram (Azone $\left.{ }^{\circledR}\right)$, isopropyl myristate and ethanol were selected as enhancers. Caffeine permeation was increased upon incorporation of all enhancers. The steady state fluxes were 05.69, 
10.13, 22.42 and $25.06 \mu \mathrm{g} / \mathrm{cm}^{2}$ per $h$ with enhancement factor equals 1.38, 2.458, 5.441 and 6.083 for 40\% EtOH, 10\% IPM, 3\% $A Z$ and $5 \% M$, respectively. Combined application of ultrasound and enhancers increased the skin permeation rate (flux) of caffeine compared with ultrasound or enhancers alone. The enhancement factor equals $1.485,4.514,18.572$, and 48.610 for $40 \% \mathrm{EOH}, 10 \%$ IPM, $3 \% A Z$ and $5 \% M$ respectively. Better effects were obtained by the combination with 5\%M. The influence of detailed conditions of ultrasound and enhancer applications on the caffeine flux was further investigated using 5\% M. Caffeine fluxes were, 95.254, 115.368 and $200.06 \mu \mathrm{g} / \mathrm{cm}^{2}$ per $h$ upon application of ultrasound for 10, 30, and 60 min respectively. The enhancement effect by this combination was increased with an increase in ultrasonic application duration, suggesting that these conditions might be used to achieve the controlled drug delivery. A pretreatment experiment with ultrasound or 5\% M was carried out, and the drug content was measured to understand the role of ultrasound in the combined effect. Pretreatment of the skin with ultrasound increased the caffeine flux, while the effect of pretreatment with $5 \% \mathrm{M}$ on the skin was similar to that of untreated skin. The results obtained suggested that simultaneous application of ultrasound and enhancers is essential to obtain the pronounced effect for transdermal as well as for topical delivery of caffeine though the skin.

\section{INTRODUCTION}

The development of a transdermal drug delivery system (TDS) is a key factor because of the high barrier properties of the stratum corneum towards drug permeation. Alterations in physicochemical properties of a drug by structural modification were attempted and characteristics of skin barrier by chemical enhancers were extensively studied ${ }^{1 \& 2}$. Iontophoresis, which delivers ionic species into the body by electric potential, may also offer the possibility of developing an intelligent TDS. This can provide pulsatile drug delivery by turning the potential on and off ${ }^{3 \& 4}$. On the other hand, skin penetration enhancement by ultrasound, has been accepted for clinical therapeutics, and is known to practically increase skin permeation of steroids and promote therapeutic efficacy for a variety of muscular and arthritic conditions ${ }^{5 \& 6}$. Trials to employ phonophoresis for a systemic drug delivery have also been performed $^{7 \& 8}$. The enhancement of skin penetration by ultrasound is found to be influenced by the alteration of the ultrasonic frequency, intensity, duration and wave mode? Therapeutic ultrasound was generally used in many cases of phonophoresis. 
The use of high ultrasound intensity for phonophoresis may result in damage to the skin or subcutaneous tissue by cavitational or thermal effect ${ }^{10}$.

A combination of low ultrasound intensity with other promoting techniques such as the use of chemical enhancers can raise the transdermal delivery rate of a drug. Several multi-component enhancement systems such as menthol and ethanol ${ }^{11}$ ultrasound and surfactant ${ }^{12}$ and D-limonene and temperature ${ }^{13}$ were studied in search of a higher enhancing effect.

Objectives in the present study were to examine the combined effect of $800 \mathrm{kHz}$ ultrasound application with an intensity of $2 \mathrm{~W} / \mathrm{cm}^{2}$ together with chemical enhancers on the skin permeation of caffeine. Menthol (M), Azone (AZ), isopropyl myristate (IPM), and ethanol (EOH) were used as enhancers. Delivery system for caffeine had been designed to enhance drug permeation through the skin for systemic use ${ }^{14}$ and to accumulate drug reservoir in the skin for local delivery in treatment of hyper-proliferative skin disease ${ }^{15}$.

\section{EXPERIMENTAL}

\section{Materials}

Caffeine (lot 310170) was purchased from Sigma chemical Co.,(St. Louis MO, USA), Hydroxy propyl methyl cellulose (HPMC) (methocel K $100 \mathrm{M}$ ), Dow Chemical company Midland, MI), Isopropyl myristate (IPM) (Merk Chemical Co., Germany). Menthol (M) (JP grade) was obtained from Adwic Pharmaceuticals, Egypt. Azone (AZ) was supplied by Nelson-Sumisho (Tokyo), other chemicals and solvents were of reagent grade and were obtained commercially.

\section{Equipment}

Previously designed modified Franz diffusion cell which allows the introduction of ultrasound probe into donor compartment is used ${ }^{16}$. Therapeutic ultrasound equipment (Shanghai, China), HPLC apparatus (model 1048, Hewlett Packard, USA). The HPLC apparatus is provided with a pump coupled with RP8 column.

\section{Methodology}

\section{Animals}

Male albino rabbits weighing 2.00-2.5 kg (7-8 weeks old), were scarified and the ear skin was taken cleaned with normal saline and the hair was removed by depilatory and freshly used in all experiments.

\section{Effect of chemical enhancers}

Each enhancer concentration was selected based on the previous studies for skin penetration enhancement: $5 \%$ $\mathrm{M}^{17}, 3 \% \mathrm{Az}^{18}, 10 \% \mathrm{IPM}^{19}$ and $40 \%$ ethanol $\mathrm{EOH}^{20}$ were used as chemical enhancers. The weight quantity of hydroxyl propyl methyl cellulose was sprinkled gently with continuous stirring on distilled water previously boiled and cooled; the stirring was continued until a thin dispersion was formed. The solution was kept over night to form a gel. The enhancer was incorporated in the gel by emulsi- 
fication with $0.5 \%$ Tween 20 with continuous stirring. One percent of the drug was incorporated with stirring with the emulsified enhancer to form emulgel.

Excised ear rabbit skin was mounted on a vertical diffusion cell (donor and receiver volume were 24.64 and $300 \mathrm{ml}$ respectively, and effective diffusion area was 24.64 $\mathrm{cm}^{2}$ ) with a water jacket connected to a water bath at $37^{\circ} \mathrm{C}$. The receiver compartment (dermis side) was filled with phosphate buffer $\mathrm{pH} 7.4$ and stirred with a star-head magnetic bar driven by a constant speed motor at $120 \mathrm{rpm}$. Ten gram of one percent caffeine gel containing each enhancer was added to the donor compartment (stratum corneum side). One $\mathrm{ml}$ sample was withdrawn from the receiver compartment at predetermined times intervals $(1,2,3,4$, $5,6,7,8,9 \mathrm{~h}$ ) to measure the caffeine concentration. One $\mathrm{ml}$ of phosphate buffer $\mathrm{pH} 7.4$ was added after sampling to keep a constant volume.

\section{Effect of combined effect of ultra- sound and chemical enhancers}

The same pervious procedure was conducted. The ultrasound probe was centrally positioned in the donor compartment. The probe was submerged into the gel in the donor compartment. Ultrasound was applied under the following conditions (intensity: $2 \mathrm{~W} / \mathrm{cm}^{2}$, mode: continuous, frequency $800 \mathrm{kHz}$, duration $60 \mathrm{~min}$, after three hours from the beginning of permeation experiment). An increase in temperature of the donor compartment during ultrasonic irradiation $\left(3-4^{\circ} \mathrm{C}\right)$ was prevented by controlling the temperature of the water bath.

Effect of ultrasonic application duration on combined application of ultrasound and $5 \% \mathrm{M}$

The ear rabbit skin was mounted on the vertical diffusion cell, as described above. To measure the effect of ultrasonic application duration, $1 \%$ caffeine gel containing $5 \% \mathrm{M}$ was added to the donor compartment, followed by ultrasonic application duration of 10,30 or 60 min after three hours from the beginning of permeation experiment and the completed.

Effect of pretreatment with ultrasound or $5 \% \mathrm{M}$ on the skin permeation of caffeine

For the pre-treatment with enhancer, $1 \%$ caffeine gel containing 5\% $\mathrm{M}$ was first applied to the donor compartment for $60 \mathrm{~min}$. The content was replaced by control gel and the ultrasound was irradiated to the compartment for $60 \mathrm{~min}$. For the pretreatment with ultrasound, $1 \%$ caffeine gel without enhancer was first added to the donor compartment, and the ultrasound was applied to the compartment for $60 \mathrm{~min}$. The donor content was then replaced with $1 \%$ caffeine gel with $5 \%$ M. Other procedures were conducted.

\section{Determination of caffeine content in the skin}

The amount of caffeine accumulated into the skin was recovered by cutting ear rabbit skin in 
to small pieces, vortex-mixed for 2 min in $10 \mathrm{ml}$ of methanol, and then subject to three sonication cycles of 30 min each in an ultrasound bath. The resulting mixture was then filtered using $0.45 \mu \mathrm{m}$ membrane and caffeine was then quantified by HPLC. The concentration of drug in skin tissues was an index of topical delivery, whereas the concentration in receptor phase was an index of transdermal delivery.

\section{Analysis}

The method of Remoussenard et $a .^{21}$, for the estimation of caffeine was adopted. $16 \%$ Methanol and $0.1 \%$ acetic acid $(84 \%)$ in water were used in HPLC as mobile phase. Samples of $0.5 \mathrm{ml}$ were mixed with $20 \mu \mathrm{L}$ of an ethanolic solution of $\beta$ hydroxy ethltheophylline solution $(100 \mu \mathrm{g} / \mathrm{ml})$ as internal standard. After centrifugation, the caffeine concentration in the supernatant was determined by (HPLC).

The steady-state flux, $J$ (given by $\mathrm{Q} / \mathrm{A} \times \mathrm{t}$, where $\mathrm{Q}$ is the amount of drug diffusing across the area $\mathrm{A}$ in time $\mathrm{t}$ ) was calculated by linear regression analysis of permeation data. The enhancing effect of enhancers on drug permeation was expressed as enhancement factor (E), calculated from the ratio $J_{b} / J_{a}$, where $J_{a}$ and $J_{b}$ are the average fluxes in the absence and in presence of enhancer, respectively.

\section{Statistical analysis}

All skin permeation experiments were repeated three times and data were expressed as the mean value \pm
S.D. Data were analyzed by one-way analysis of variance (ANOVA). A multiple comparison test was used to compare different formulations, and a $\mathrm{P}$-value of $\leq 0.05$ was considered to be significant.

\section{RESULTS AND DISCUSSION}

\section{Effect of chemical enhancers on caffeine permeation}

Table 1 shows caffeine fluxes at a steady state $(6-8 \mathrm{~h})$ after application of gels containing each enhancer. A permeation experiment using $1 \%$ caffeine and $1 \%$ Tween 20 was carried out as a control (without enhancer), since the influence of Tween 20 on the skin permeation of drugs can be ignored, compared with general enhancers ${ }^{22}$.

Caffeine permeation was found to increase upon incorporation of the selected enhancers. The steady state fluxes were $05.69,10.13,22.42$ and $25.06 \mu \mathrm{g} / \mathrm{cm}^{2}$ per h with enhancement factor equals $1.38,2.458,5.441$ and 6.083 for $40 \%$ EOH, 10\%IPM, 3\% $\mathrm{AZ}$ and 5\% M respectively. Ethanol has been used to enhance the flux of levonorgestrel, estradiol, hydrocortisone and 5-fluorouracil through rat $\operatorname{skin}^{23}$. However, the lower enhancing effect might be due to higher ethanol levels (40\%) which cause dehydration of the biological membrane and reduced permeation across the tissue ${ }^{24}$, while, 10\% IPM enhances permeation by penetrating into the stratum corneum and increasing the lipid fluidity by disruption of lipid packing. In addition, it influences partitioning 
between vehicle and skin by solubilization effects ${ }^{25}$. Azone probably exerts its penetration enhancing effects through interactions with the lipid domains of the stratum corneum.

The higher flux value (25.06 $\mu \mathrm{g} / \mathrm{cm}^{2}$ per h) with about 6-fold increase in caffeine permeation was achieved with $5 \% \mathrm{M}$. Menthol is a monocyclic terpene with a pleasant taste and odor. It is widely used as a flavoring agent in oral dosage forms and as a fragrance with a mild antiprutitic effect in topical formulations. Due to the pleasant taste associated with menthol, its use in transdermal drug delivery system may increase patient acceptability. The highest enhancing activity of menthol is due to its disruption to the bilayer of $\mathrm{SC}$ and form pools in the $\mathrm{SC}^{2}$.

\section{Combined effect of ultrasound and chemical enhancers}

Steady-state fluxes for control gel without and with ultrasound were $4.12 \pm 0.22$ and $6.42 .00 \pm 0.90 \mu \mathrm{g} / \mathrm{cm}^{2}$ per $\mathrm{h}$ respectively (Tables $1 \& 2$ ). The ultrasound effect on control gel was only 1.55 -fold. The enhancement factor (E) for each enhancer with or without ultrasound was also revealed in Figure 1.

Application of 5\% $\mathrm{M}$ alone induced about 6-fold increase in caffeine permeability, while combined application of ultrasound induced 48.6 enhancements. These results was in agreement with Mitragotri et al. ${ }^{26}$.

Table 1: Caffeine fluxes at a steady state $(6-8 \mathrm{~h})$ and the enhancement factor (E) using $1 \%$ caffeine gels containing different enhancers.

\begin{tabular}{|l|c|c||}
\hline \multicolumn{1}{|c|}{ Gel composition } & $\begin{array}{c}\text { Flux (Jss) } \\
\left(\mu \mathrm{g} / \mathrm{cm}^{2} \text { per } \mathrm{h}\right)\end{array}$ & $\begin{array}{c}\text { Enhancement } \\
\text { factor (E) }\end{array}$ \\
\hline $\begin{array}{l}\text { 1\% caffeine \& 1\% Tween 20 } \\
\text { (Control gel) }\end{array}$ & \multicolumn{2}{|c|}{$4.12 \pm 0.22$} \\
\hline Enhancers & $25.06 \pm 1.21$ & 6.083 \\
\hline Gel with 5\% M & $22.42 \pm 1.81$ & 5.441 \\
\hline Gel with 3\% AZ & $10.13 \pm 0.58$ & 2.458 \\
\hline Gel with 10\% IPM & $05.69 \pm 0.32$ & 1.380 \\
\hline Gel with 40\% EOH &
\end{tabular}

Each value represents the mean \pm S.D of three experiments. 
Table 2: Caffeine fluxes at a steady state (6-8 h) and the enhancement factor (E) after combined application of $1 \%$ caffeine gels containing different enhancers and ultrasound.

\begin{tabular}{||l|c|c||}
\hline \multicolumn{1}{|c|}{ Gel composition } & \multicolumn{1}{c|}{$\begin{array}{c}\text { Flux (J ss) } \\
\left(\mu \mathrm{g} / \mathrm{cm}^{2} \text { per } \mathrm{h}\right)\end{array}$} & $\begin{array}{c}\text { Enhancement } \\
\text { factor }(\mathrm{E})\end{array}$ \\
\hline $\begin{array}{l}\text { 1\% caffeine \& 1\% Tween 20 } \\
\text { (Control gel) }\end{array}$ & $6.42 .00 \pm 0.90$ & 1.550 \\
\hline Enhancers & $200.31 \pm 10.53$ & 48.610 \\
\hline Gel with 5\% M & $76.52 \pm 8.53$ & 18.572 \\
\hline Gel with 3\% AZ & $18.60 \pm 1.08$ & 4.514 \\
\hline Gel with 10\% IPM & $6.12 \pm 0.27$ & 1.485 \\
\hline Gel with 40\% EOH &
\end{tabular}

Each value represents the mean \pm SD of three experiments.

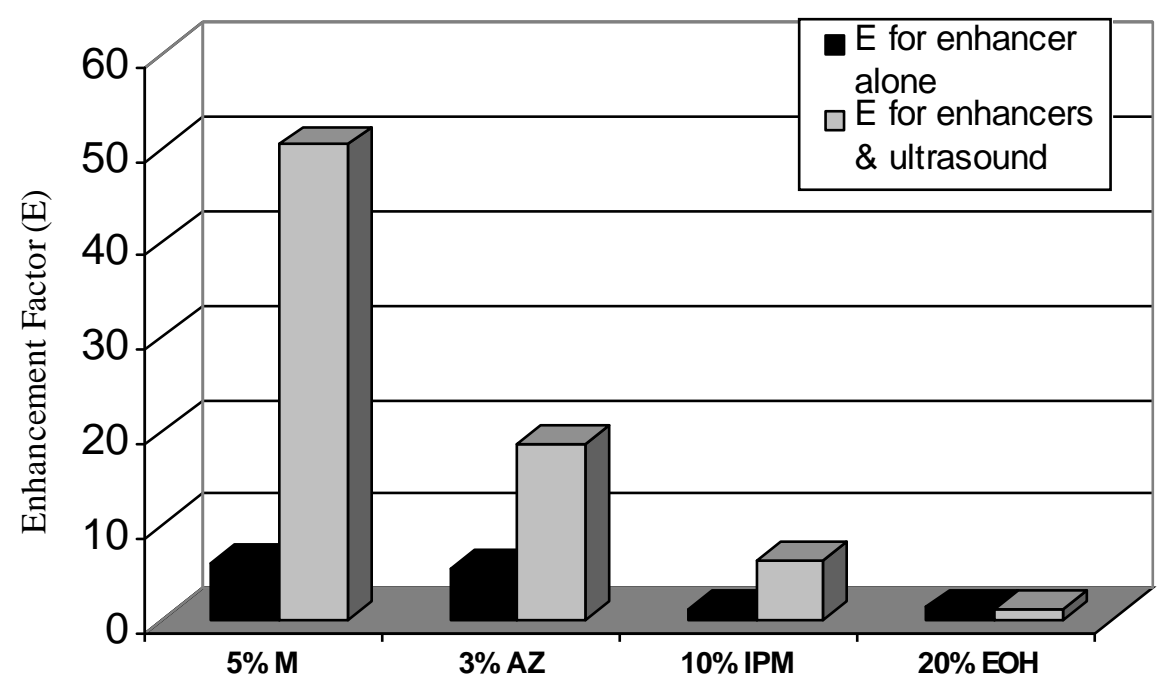

Fig. 1: Effect of enhancers and ultrasound on caffeine permeation through the skin.

Various possible mechanisms of synergistic effect of ultrasound and enhancers were indicated including enhanced delivery and dispersion of the enhancer in the skin due to ultrasound ${ }^{13}$. In addition to increasing transdermal transport, a combination of ultrasound with other enhancers also reduces the severity of the enhancers required to achieve the 
desirable drug flux. Hence, a combination of ultrasound with other enhancers may not only increase the total enhancement, but can also increase the safety by reducing the strength of individual enhancers.

\section{Effect of the duration of ultrasonic application}

Figure 2 shows the influence of the duration of ultrasonic application on the combined effect of ultrasound and $5 \% \mathrm{M}$. Caffeine flux with the combined application was higher than that with 5\% M.Caffeine fluxes were, 95.254115 .368 and $200.06 \mu \mathrm{g} / \mathrm{cm}^{2}$ per $\mathrm{h}$ upon application of ultrasound for 10,30 , and $60 \mathrm{~min}$ respectively. Caffeine permeation increased with increases in the duration from 10, 30 and 60 min showing that regulation of these factors in the vehicle may aid in controlled drug delivery with a higher enhancement level than that of ultrasound alone.

\section{Effect of pretreatment with 5\% M or ultrasound on the skin permea- tion of caffeine}

Figure 3 demonstrates the time courses of caffeine flux from pretreatment with $5 \% \mathrm{M}$ or ultrasound. Caffeine flux was 25.23 and $26.25 \mu \mathrm{g} / \mathrm{cm}^{2}$ per $\mathrm{h}$ for the skin without treatment and $5 \% \mathrm{M}$ pretreated skin respectively. The difference was not statistically significant. On the other hand, one hour application of ultrasound to the skin before permeability experiment induced a marked increase in caffeine flux $\left(200.06 \mu \mathrm{g} / \mathrm{cm}^{2}\right.$ per $\left.\mathrm{h}\right)$. In this method, a short application of ultrasound is used to permeabilize skin prior to drug delivery. The skin remains in a state of high permeability for several hours. Drugs can be delivered through permeabilized skin during this period. In this approach, the patient does not need to wear the ultrasound device ${ }^{24}$.

\section{Determination of caffeine content in the skin}

Caffeine content on the skin layers is presented in Table 3. The drug content was 1.23 and $0.10(\mathrm{mg} / \mathrm{g}$ skin) for control gel with and without application of ultrasound respectively. The caffeine content was 15.25 and 7.84 (mg/g skin) for gel with $5 \% \mathrm{M}$ with and without ultrasound application respectively. These results were in agreement with that reported by Williams and Barry $(2004)^{24}$ who reported that skin penetration enhancement by monoterpenes occurred through an increase in diffusivity of polar compounds in the stratum corneum.

Also, menthol increased the migration of aqueous donor solvent into the stratum corneum ${ }^{20}$. Combination of $5 \% \mathrm{M}$ and ultrasound might offer a synergistic effect for caffeine accumulation in the skin layers as the content was $15.25 \mathrm{mg} / \mathrm{g}$ of the skin. Therefore, ultrasound multiplies the enhancing action of menthol in such a way as to increase the migration of aqueous donor solvent into the stratum corneum. This approach can be effective for topical treatment of hyperproliferative skin diseases. 


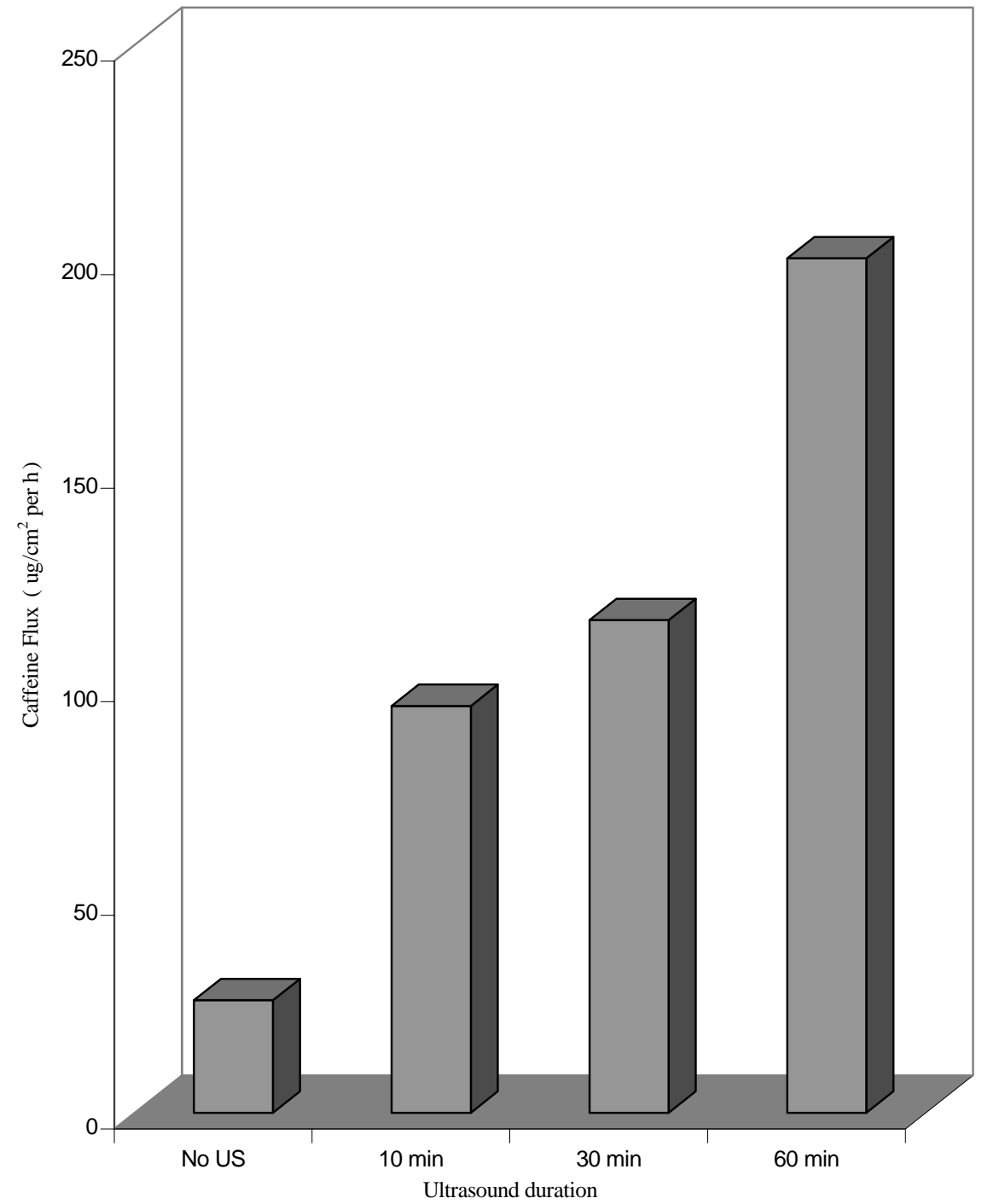

Fig. 2: effect of sonophoresis duration on caffeine permeation from $5 \%$ methol gel through the skin. 


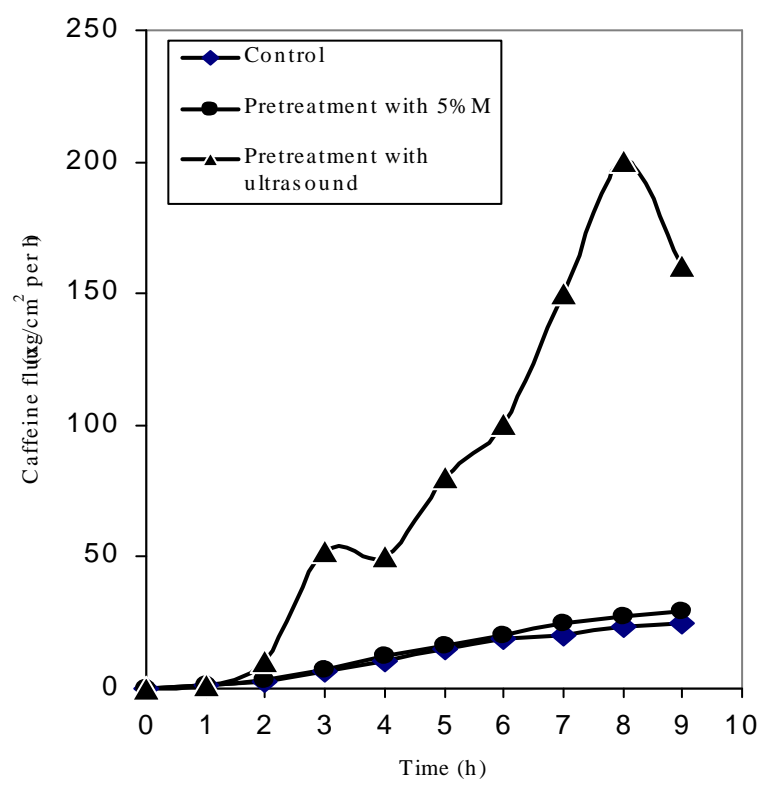

Fig. 3: Time course of caffeine flux in the pretreatment experiment with $5 \% \mathrm{M}$ or ultrasound.

Table 3: Caffeine content in the skin tissues after permeability experiments with $1 \%$ caffeine gel containing $5 \% \mathrm{M}$ with and without ultrasound.

\begin{tabular}{||l|c|c|}
\hline \multirow{2}{*}{ Gel composition } & \multicolumn{2}{|c|}{$\begin{array}{c}\text { Caffeine content } \\
\text { (mg/g skin) }\end{array}$} \\
\cline { 2 - 3 } & With ultrasound & Without ultrasound \\
\hline $\begin{array}{l}\text { 1\% caffeine \& 1\% Tween } \\
\text { (Control gel) }\end{array}$ & $1.23 \pm 0.320$ & $0.10 \pm 0.02$ \\
\hline Gel with $5 \% \mathrm{M}$ & $15.25 \pm 2.02$ & $7.84 \pm 1.02$ \\
\hline
\end{tabular}

Each value represents the mean \pm SD of three experiments.

\section{Conclusion}

The simultaneous application of ultrasound and chemical enhancers was found to achieve a great increase in skin penetration of caffeine. When enhanced drug delivery by ultrasound and chemical enhancers is anticipated, selection of the best combination becomes the key to assuring high ultrasonic enhancement, because the skin permeation rate of a drug is influenced by ultrasonic conditions such as duration of application. 
Combinations of ultrasound with 5\% menthol may be useful for effective skin delivery of caffeine and hence obtaining a more pronounced therapeutic effect.

\section{REFERENCES}

1- K. A. Waiters and J. Hadgraft, Pharmaceutical Skin Penetration Enhancement, Marcel Dekker, New York (1993).

2- D. Mitragotri, J. Ray, H. Farrell, B. Tang, J. Yu, D. Kost, R. Blankschtein and Langer, J. Pharm. Sci., 89, 892 (2000).

3- M. Nakakura, M. Terajima, Y. Kato, E. Hayakawa, K. Ito and T. Kuroda, J. Drug Targeting, 2, 487 (1995).

4- R. M. Brand and R. H. Guy, J. Control. Release, 33, 385 (1995).

5- J. C. McElnay, T. A Kennedy and R. Harland, Int. J. Pharm., 40, 105 (1987)

6- G. Merriono, Y. N. Kalia, M. B. Delgado-Charro, R. Potts and R. H. Guy, J. Control. Release, 88, 85 (2003).

7- S. Mitragotri, D. Blankschtein and R. Langer, Science, 269, 850 (1995).

8- A. Joshi, J. Raje, J. Control. Release, 83, 13 (2002).

9- J. C. McElnay, H. A. E Benson, J. Hadgraft and T. M Murphy, The use of ultrasound in skin penetration enhancement. In Waiters, K.A. and Hadgraft, J. (Eds.), Pharmaceutical Skin Penetration Enhancement, Marcel Dekker, New York, 1993, pp. 293-309.
10- K. S. Suslick, Ultrasound: It's Chemical, Physical, and Biological Effects, VCH, New York (1988).

11- Y. Morimoto, K. Sugibayashi, D. Kobayashi, H. Shoji, J. Yamazaki and M. Kimura, Int. J. Pharm., 91, 9 (1993).

12- A. Tezel, A. Sanders, J. Tuchscherer and S. Mitragotri, J. Pharm. Sci., 91, 91 (2001).

13- M. Amato, M. Isenschmid and P. Huppi, Eur. J. Pediat., 150, 592 (1991).

14- N. Ohara, K. Takayama, Y. Machida and T. Nagai, Int. J. Pharm., 105, 31 (1994).

15- E. Touitou, F. Levi-Schaffer, Shaco-Ezra, N. R. Ben Yossef and B. Fabin, ibid., 70, 159 (1991).

16- F. Sakr, E. Ramadan and Th. Borg, Mans J. Pharm. Sci., 15, 110 (1999).

17- K. Sugibayashi, D. Kobayashi, E. Nakagaki, T. Hatanaka, N. Inoue, M. Kusumi, M. Kobayashi,, M. Kimura and Y. Morimoto, Int. J. Pharm., 113, 189 (1995)

18- Y. Morimoto, T. Atanaka, M. Oguchi,, K. Sugibayashi, M. Kobayashi and M. Kimura, S.T.P. Pharm. Sci., 2, 253 (1992).

19- M. Okumura, Y. Nakamori, K. Sugibayashi and Y. Morimoto, Drug Des. Del., 7, 147 (1991).

20- D. Kobayashi, T. Matsuzawa, K. Sugibayashi, Y. Morimoto and M. Kimura, Pharm. Res., 11, 96 (1994). 
21- O. Remoussenard, P. Treffel., Y.Bechtel and P. Agacche, J. Pharm. Sci., 82, 1099 (1993).

22- Y. Morimoto, K. Sugibayashi, K. Hosoya and W. I Higuchi, Int. J. Pharm., 32, 31 (1986).

23- D. Friend, P. Catz, J. Heller, J. Reid and R. Baker, J. Control. Release 7, 243 (1988).
24- A. C. Williams and B.W. Barry, Adv. Drug Deliv. Rev., 56, 603 (2004).

25- K. Sate, K. Sugibayashi and Y. Morimoto, Int. J. Pharm., 43, 31 (1988).

26- S. Mitragotri, and S. Kost, Adv. Drug Deliv. Rev. 56, 589 (2004). 